\title{
Alguns aspectos socioeconômicos relacionados a parasitoses intestinais e avaliação de uma intervenção educativa em escolares de Estiva Gerbi, SP
}

\author{
Some socioeconomic aspects related to intestinal parasitosis \\ and evaluation of an educational intervention in scholars \\ from Estiva Gerbi, SP
}

Glauco Rogério Ferreira ${ }^{1}$ e Carlos Fernando Salgueirosa Andrade ${ }^{2}$

\begin{abstract}
RESUM0
Verificou-se entre os escolares atendidos pela Secretaria Municipal de Educação de Estiva Gerbi-SP, a prevalência de $11,5 \%$ de enteroparasitas, que puderam ser relacionados a aspectos socioeconômicos. Realizou-se então uma oficina de trabalho sobre parasitas intestinais no sentido de informar e discutir sobre esta questão, complementando o tratamento médico. Em especial, foi envolvida a Escola Alaíde R. Batista que atende crianças até 4 anos de idade, e que havia mostrado a maior (23,5\%) taxa de parasitismo. Uma avaliação dois anos após a oficina de trabalho mostrou que a prevalência de enteroparasitas nos escolares tratados foi reduzida, e era de 6,6\%. Na escola como um todo, devido ao ingresso de novos estudantes, a prevalência era ainda de 23,3\%. Concluiu-se que atitudes educativas são válidas, mas precisam ser integradas a um processo contínuo de educação e controle das enteroparasitoses.
\end{abstract}

Palavras-chaves: Enteroparasitoses. Oficina de trabalho. Prevalência. Estudantes.

\begin{abstract}
Among the students attended by the City Education Secretary of Estiva Gerbi, 11.5\% presented enteroparasites. This prevalence could be related to socioeconomic aspects. Accordingly we conducted a workshop on intestinal parasites with the objective of informing and discussing related aspects in combination with medical treatment. Students were evaluated from the Alaíde R. Batista School, which attends children up to 4 years old and had the highest (23.5\%) level of parasitism. Reevaluation two years after the workshop showed that the prevalence of enteroparasites in the treated students had decreased to $6.6 \%$, but considering the school as a whole the prevalence was still $23.3 \%$, due to the enrolling of new students. It was concluded that educational programs are only effective when integrated to a complete and continuous process of education, control and eradication of enteroparasitosis.
\end{abstract}

Key-words: Enteroparasitosis. Workshop. Prevalence. Students.

0 parasitismo intestinal ainda se constitui um dos mais sérios problemas de Saúde Pública no Brasil, principalmente pela sua correlação com o grau de desnutrição das populações, afetando especialmente 0 desenvolvimento físico, psicossomático e social de escolares.

Segundo Santos cols ${ }^{14}$, embora haja uma vasta literatura sobre a importância das enteroparasitoses para a Saúde
Pública, e especialmente, em relação a escolares, pouca atenção tem sido dada ao assunto, nos programas de formação de educadores.

Objetivou-se com este trabalho conhecer a prevalência desses parasitas intestinais, os principais aspectos epidemiológicos envolvidos e sua relação a aspectos sócio culturais e avaliar uma intervenção educativa sobre a questão das parasitoses intestinais.

1. Departamento de Parasitologia do Instituto de Biologia da Universidade Estadual de Campinas, Campinas, SP. 2. Departamento de Zoologia do Instituto de Biologia da Universidade Estadual de Campinas, Campinas, SP.

Endereço para correspondência: Dr. Glauco Rogério Ferreira. R. Paula Bueno 672, Centro, 13840-040 Moji Guaçu, SP.

Tel: 5519 3861-7793.

e-mail: glaucorf@ terra.com.br, cfeandra@ unicamp.br

Recebido para publicação em 27/6/2004

Aceito em 6/6/2005 


\section{MATERIAL E MÉTODOS}

A entrega dos coletores de fezes foi acompanhada de uma ficha padrão para preenchimento pelos pais ou responsáveis, para 0 levantamento dos aspectos socioeconômicos dos alunos e suas famílias. Para determinar a existência de relação de dependência entre 0 parasitismo e os aspectos sócios ambientais foi utilizado 0 teste do $\chi^{2}$, para 0 nível de significância de $5 \%$.

0 presente projeto foi autorizado pelo Comitê de Ética em Pesquisa Médica da Universidade Estadual de Campinas (processo no 470/01), e teve prévia autorização dos pais ou responsáveis pelos escolares envolvidos.

No período de estudo, que foi de 2000 a 2002, a Prefeitura Municipal de Estiva Gerbi atendia em sua rede de ensino em torno de 1.000 escolares que variavam de 0 a 7 anos de idade, distribuídos em 8 escolas, sendo 2 creches, 4 Escolas Municipais de Ensino Infantil (EMEIs), uma Escola Municipal de Ensino Fundamental (EMEF) e a Associação de Pais e Amigos dos Excepcionais (APAE) . No ano de 2000, após realização do levantamento parasitológico, as crianças positivas foram encaminhadas ao posto de saúde municipal para tratamento. Em seguida, foi estruturada uma oficina de trabalho para os pais e alunos das EMEIs, creches e da EMEF. Nessa oficina de trabalho foram apresentados conhecimentos sobre os vários tipos de parasitas e comensais comumente encontrados, sobre os ciclos biológicos dos mesmos, as formas de transmissão e métodos de profilaxia.

Os participantes foram convidados a seguir ao pátio do Centro Cultural, onde foram aplicadas duas dinâmicas: 1. lavagem de verduras e 2. lavagem das mãos.

A dinâmica de lavagem de verduras foi montada de forma a se poder verificar a eficácia na remoção de um marcador invisível ( tinta invisível fluorescente Meyerman). A verdura escolhida foi a couve-manteiga, por ser encontrada na maioria das residências que possuem horta, pela alta taxa de consumo da população e ainda por ser resistente à lavagem e fácil marcação com tinta fluorescente. Usou-se nessa avaliação água sanitária ( teor de cloro de $2 \%$ a 2,5\%), vasilha, colher de café e lâmpada de luz negra.

Comparou-se então os resultados obtidos entre a lavagem demonstrada por eles e pelo procedimento indicado como correto. Essa comparação foi feita expondo-se as verduras à lâmpada de luz negra, que evidencia os contrastes da tinta fluorescente invisível à luz natural.

A dinâmica de lavagem das mãos foi realizada da seguinte maneira: 1. As pessoas que participaram receberam uma aplicação de tinta hidrossolúvel guache preta nas mãos; 2 . Em seguida, tiveram os olhos vendados e foram colocados à frente de uma torneira com água corrente e sabão para lavarem as mãos. Depois que as pessoas lavaram as mãos, foram retiradas as vendas para que avaliassem se foram bem lavadas. Aavaliação foi determinada em três graus: mãos bem, medianamente e mal lavadas. Depois foi aplicado um questionário para avaliar os conhecimentos específicos adquiridos.

№ ano de 2002, foi decidido pela Administração Municipal de Estiva Gerbi que o segundo exame coprológico, seria realizado nas crianças da CECI Alaíde R. Batista, devido a maior taxa de parasitismo encontrada. ACECI Alaíde R. Batista atende em torno de 60 escolares que estão na faixa etária dos 0 a 6 anos de idade, vindas de todos os bairros da cidade, variando, portanto, quanto aos níveis sociais.

Os métodos adotados para os exames coprológicos foram: 0 método direto, sedimentação espontânea, Ritchie e de Faust e colaboradores.

\section{RESULTADOS}

A amostra dos escolares no presente inquérito foi de 930 alunos. Destes, 96,7\% atenderam à solicitação de colheita de material para exame de fezes. Para os totais realizados, apenas $11,5 \%$ apresentaram positividade para pelo menos um parasita ou comensal intestinal.

Verificamos no CECI Alaíde R. Batista a maior taxa de parasitismo, com 23,5\% dos escolares infectados, seguindo gradativamente menores valores nas outras escolas, pela ordem: EMEI Alzira Oliveira Correa (15,7\%); EMEI Maria de Lourdes S. Abreu (14,7\%); APAE (12,8\%); Escola Municipal de Ensino Fundamental (EMEF) Adélia Caleffi Gerbi (12,1\%); Casa da Criança Adélia Caleffi Gerbi (6,1\%), EMEI Milton Franco de Faria $(4,1 \%)$ e EMEI João Francisco de Lima (2,6\%).

Entre os estudantes, a maior intensidade de parasitismo verificada foi representada pelo protozoário comensal Entamoeba coli, com 47 escolares parasitados ( prevalência = 5,2\%). Eem seguida, ocorreu o protozoário Giardia duodenalis, com 45 (5\%) escolares parasitados. Pela ordem, apareceu ainda 0 helminto Ascaris lumbricoides, com 14 (1,5\%) escolares parasitados, e 0 protozoário comensal Endolimax nana, com $8(0,8 \%)$ escolares parasitados. Os demais representam a menor parte do parasitismo intestinal, revelando contudo a presença de um amplo espectro de parasitoses intestinais. Assim, aparecem nas amostras os helmintos Enterobius vermicularis $(0,2 \%)$, Hymenolepis nana, $(0,1 \%)$, Trichuris trichiura (0,1\%) e os ancilostomatídeos: os helmintos Ancylostoma duodenalis e Necator americanus, ambos com provável incidência na região, mas indistinguíveis no exame de fezes e com equivalência clínica e epidemiológica $(0,1 \%)$. Os resultados foram agrupados para as diversas escolas e em relação ao número de casos positivos.

0 poliparasitismo apresentou $12,5 \%$ de freqüência com relação ao número de casos positivos. Oito $(7,2 \%)$ escolares apresentavam dois parasitas ou comensais simultaneamente, três $(2,7 \%)$ escolares apresentavam três parasitas/comensais e um $(0,9 \%)$ escolar apresentava quatro parasitas/comensais. 0 número médio de espécies presentes por escolares foi de 1,1 parasita por escolar contaminado, e tal fato não indica que o poliparasitismo seja freqüente, como foi verificado. Tais valores diferem dos apresentados para algumas cidades do Brasil, e do Estado de São Paulo ${ }^{1}$, respectivamente com $49 \%$ e $65,2 \%$ de monoparasitismo e $51 \%$ e $34,8 \%$ de poliparasitismo, salientando-se que na área rural o poliparasitismo continua mais acentuado. 
Foram analisados 850 questionários, relativos a 900 escolares, uma vez que foi levado em consideração apenas um questionário por família.

Os resultados da avaliação das condições socioeconômicas e culturais mostraram que quanto à Localização da residência, A zona rural do município era habitada por 15,4\%. Ao aplicar 0 teste $\chi^{2}$ notou-se uma diferença significativa $(p=0,00001)$ para uma maior ocorrência de enteroparasitas entre os residentes na zona rural. Isto pode ser explicado devido à situação mais precária do saneamento básico encontrado na maioria das residências rurais do município.

Para as variáveis material de construção, material de revestimento, número de cômodos, águas e reservatórios, águas servidas e animais domésticos, não se obteve uma diferença estatísticas significativa.

Para a questão localização da privada, verificou-se que na maioria ( $73,9 \%)$ das casas a privada era dentro da residência, sendo que $97,8 \%$ destas possuía descarga, ou seja quase a sua totalidade. Também foi observada uma diferença significativa $(\mathrm{p}=0,008)$ para a localização dos banheiros nas residências, sendo maior o número de casos positivos para enteroparasitas quando os banheiros eram localizados fora das residências. Para Chieffi cols ${ }^{3}$, algumas condições de promiscuidade proporcionadas por banheiros e dormitórios coletivos favoreceram a transmissão de T. trichiurus e $\mathrm{H}$. nana, e ainda, 0 encontro de ovos de Ascaris lumbricóides, Taenia sp e ancilostomatídeos nos banheiros coletivos de um orfanato em Londrina, PR aonde foi feito o estudo.

A maioria (86,7\%) das casas possuía caixa d'água, e apenas uma pequena (13\%) parcela utilizava ligação direta da rede pública de abastecimento. Em 98,6\% das residências, a caixa d'água era mantida tampada. Uma proporção de 1,4\% não sabia informar se a mesma estava tampada, servindo nesse último caso como possível criadouro de mosquitos, e estando sujeitas a albergar outros animais e insetos. Para essa variável (possuir caixa d'água) obteve-se uma diferença significativa ( $p=0,01)$, com mais casos de escolares positivos para as residências sem caixa d'água, e aquelas cujos moradores entrevistados não sabiam se a caixa era mantida tampada.

A maior incidência de animais sinantrópicos relatada nos questionários foi para dípteros ( mosquitos) com freqüência de $742(82,4 \%)$ casos relatados, e talvez este fato se deva à existência de dois córregos e um pequeno rio que atravessam o município em toda sua extensão, facilitando a reprodução destes insetos. As baratas constituem um tormento para a população local, pois conforme citado, ocorreram em 70,1\% das residências do município. As moscas domésticas ocorreram em $60,7 \%$ das casas sendo outro animal a ser levado em consideração. Tais animais sinantrópicos representam um sério risco à população, pois servem de vetores mecânicos dos enteroparasitas, devido aos seus hábitos. Obteve-se uma diferença significativa $(p=0,0006)$ para animais sinantrópicos com relação ao número de casos positivos para parasitas intestinais.

Das 200 pessoas que compareceram a oficina de trabalho, no ano 2000, 40\% participaram voluntariamente da dinâmica de Lavagem de Verduras. Dos 40\%, apenas 6,2\% lavaram as verduras satisfatoriamente, mas sem as colocar na solução desinfectante que estava disponível, resultando ainda na folhas lavadas algum contraste da tinta invisível fluorescente na presença da luz negra. Após terem aprendido a maneira correta de lavagem e descontaminação, todos constataram a eficácia do método: 15\% apresentaram uma lavagem de qualidade Média (quantidade mínima do marcador fluorescente) e $85 \%$ demonstraram uma lavagem Boa (ausência do marcador fluorescente) das verduras.

Na dinâmica da Lavagem das Mãos, participaram voluntariamente 50 pessoas, obtendo-se os seguintes resultados: $46 \%$ praticaram uma boa lavagem das mãos; 30\% fizeram uma lavagem mediana de mãos; e 24\% tiveram mãos mal lavadas. Consideramos esse último valor elevado, aproveitando para a demonstração da necessidade de se lavar adequadamente as mãos com sabão e água corrente. A discussão é baseada em Campos ${ }^{1}$, e sua indicação de que a higiene das mãos é um importante fator que predispõe as pessoas, principalmente crianças, à infecção por enteroparasitas. E justifica-se esta dinâmica, de valorização da higidez das mãos, no sentido do trabalho de Pedrazzani cols ${ }^{11}$, que interpõem a importância de práticas educativas como melhores fomentadoras de informações e capacitação formadora de opiniões.

Dos 200 participantes da oficina de trabalho, 75\% (150 escolares e pais/responsáveis) responderam ao questionário de conhecimentos sobre as parasitoses, onde foram obtidos resultados significativos, notando-se que houve ampla assimilação dos conhecimentos e mudanças nos hábitos e conceitos apontados anteriormente.

\section{DISCUSSÃ0}

0 resultado dos exames coprológicos em 2002 indicou que as crianças da CECI Alaíde R. Batista apresentavam uma taxa de parasitismo de 23,3\%, sendo maior o número de casos positivos para 0 comensal E. coli (prevalência de 13,3\%), seguido da G. duodenalis (10\%) e de ALumbricoides (6,6\%). № trabalho anteriormente realizado no ano 2000 a taxa geral de parasitismo era praticamente a mesma ( $23,5 \%)$, no entanto E. coli tinha uma prevalência menor $(6,9 \%)$, G. duodenalis era também menos comum (14,7\% de prevalência) e A. lumbricoides com $5,8 \%$.

Desde a avaliação no trabalho anteriormente realizado, a CECI Alaíde Batista mudou sua sede que estava localizada em área central da cidade para um bairro recém criado em 2001 onde não haviam vias asfaltadas. Esta mudança de localização e a contínua troca de funcionários parecem ter sido importantes fatores para a manutenção das taxas de parasitismo nas crianças. Franco \& Cordeir $0^{6}$, por exemplo, relatam que atendentes inexperientes colocam os escolares em riscos de infecção sendo um fator decisivo para a manutenção das taxas de parasitismo. Coelho cols², analisaram a presença de ovos e larvas de helmintos em elementos de sanitários, alcançando uma prevalência de $15,4 \%$ das crianças analisadas, concluindo que a presença de ovos de helmintos nos sanitários indica a possibilidade de 
transmissão das enteroparasitoses, principalmente em sanitários coletivos, como o caso de escolas e creches. № que diz respeito à rotatividade de funcionários, isto se resolveria a partir da efetivação do quadro dos mesmos, e posterior treinamento, diminuindo-se os riscos de infecção. Com a mudança da CECI Alaíde R. Batista para sede própria foi ainda ampliado o número de vagas, que antes era de 30 escolares, passou para 60 escolares. Machado cols $^{10}$, avaliaram a prevalência de parasitoses intestinais em escolares da rede pública e privada, e observaram que as freqüências foram semelhantes entre as creches, porém maiores nas escolas públicas em relação às particulares, apontando os indicadores de nível socioeconômico, de escolaridade e de saneamento como determinantes para adquirir as parasitoses intestinais. No presente estudo, das crianças que se apresentaram positivas apenas 6,6\% ( 4 escolares) eram crianças que já eram atendidas pela CECI, configurando portanto casos de reinfecção. Estes 4 escolares pertencem à mesma família, sendo sua reinfecção possivelmente originada de outros membros parasitados daquela residência. Moretti cols ${ }^{12}$ já indicaram que as enteroparasitoses têm sua transmissão facilitada quando há o contato pessoa-pessoa, como também 0 uso de quartos e banheiros coletivos propiciam a transmissão dos parasitos intestinais.

Os outros escolares positivos começaram a ser atendidos pela CECI no ano de 2002, portanto, os pais responsáveis não participaram da oficina de trabalho realizado em junho de 2001 demonstrando a importância da inclusão de práticas educacionais em saúde com a participação ativa dos envolvidos.

Concluiu-se que escolares de Estiva Gerbi apresentavam percentuais e diversidade de parasitismo comparáveis às demais regiões de interior do Estado de São Paul0 ${ }^{45671315}$.

A situação ímpar do município apresentar boas condições de saneamento nos mostra que ainda assim são encontradas parasitoses, devido à falta de orientação e higiene por parte da população. Há necessidade de dedicar mais atenção e planejamento estratégico dos dirigentes para captação de recursos financeiros a fim de implementar as ações que viabilizem 0 controle das parasitoses no município.

Indica-se que as práticas educacionais quando bem aplicadas levam as pessoas a adquirirem os conhecimentos para prevenção de parasitoses, alcançando objetivos propostos e evidenciando o valor da orientação pedagógica para a conscientização da população.

\section{REFERÊNCIAS BIBLIOGRÁFICAS}

1. Campos R, Briques W. Levantamento multicêntrico de parasitoses intestinais no Brasil, Rhodia, 1993.

2. Coelho LMPS, Sobrinho TA, Oliveira SM, Ikegami MT, Yoshizumi AM, Nakamoto YK, Brotto AS, Felberg S, Maiorano MR Ovos e larvas de helmintos nos sanitários de pré-escolas municipais de Sorocaba, SP e suas frequêencias nas fezes das crianças. Revista da Sociedade Brasileira de Medicina Tropical 32:647-652, 1999.

3. Chieffi PP, Moretti IG, Folzer ACM, Nakagawa E, Gomes AC. Contribuição ao estudo da história natural de enteroparasitoses em uma comunidade fechada. Revista da Sociedade Brasileira de Medicina Tropical 8:87-91, 1974.

4. Chieffi PP, Waldman EA, Dias RSDS, Torres DMAGV, Chimara R, Mizumoto LC, Silva AMA, Uehara M. Enteroparasitoses no município de Guarulhos, SP. 1. Prevalência de infecção entre escolares residentes no bairro de Taboão, em junho de 1984. Revista do Instituo Adolfo Lutz 48:75-85, 1988.

5. Ferreira UM, Ferreira CS, Monteiro CA Tendência secular das parasitoses intestinais na infância na cidade de São Paulo(1984-1996). Revista de Saúde Pública 34:73-82, 2000.

6. Franco RMB, Cordeiro NS. Giardiose e Criptosporidiose em creches do município de Campinas, SP. Revista da Sociedade Brasileira de Medicina Tropical 29:585-591, 1996.

7. Gioia I. Levantamento eco-parasitológico da população residente na Fazenda Intervales, SP. 1995. Tese de Doutorado, Faculdade de Saúde Pública, Universidade de São Paulo, SP, São Paulo 1995.

8. Hoffman WA, Pons JA, Janer JL. The sedimentation-concentration method in schistosomiasis mansoni. Puerto Rico Journal Public Health 9:283-298, 1934.

9. Instituto Brasileiro de Geografia e Estatística. X Recenseamento Geral do Brasil - 1996, São Paulo 1:467, 1997.

10. Machado RC, Marcari EL, Cristante SFV, Carareto CMA, Giardíase e Helmintíases em crianças de creches e escolas de $1 \underline{0}$ e $2^{\circ}$ graus (públicas e privadas) da cidade de Mirassol (SP, Brasil). Revista da Sociedade Brasileira de Medicina Tropical 32:697-704, 1999.

11. Mello DA, Pripas S, Fucci M, Santoro MC, Pedrazzani ES. Helmintoses intestinais. I. Conhecimentos, atitudes e percepção da população. Revista de Saúde Pública 22:140-149, 1988.

12. Moretti IG, Chieffi PP, Nakagawa E, Gomes C, Foizer ACM. Contribuição ao estudo da história natural de enteroparasitoses em uma comunidade fechada. Revista da Sociedade Brasileira de Medicina Tropical 3:41-43, 1974.

13. Pedrazzani ES, Mello DA, Pizzigatti CP, Barbosa CAA. Aspectos educacionais da intervenção em helmintoses intestinais, no subdistrito de Santa Eudóxia, município de São Carlos - SP. Caderno de Saúde Pública 6:74-85, 1990.

14. Santos MG, Massara Cl, Morais GS. Conhecimentos Sobre Helmintoses Intestinais De Crianças De Uma Escola De Minas Gerais, Revista Brasileira de Programa de Ciências, 42:188-194, 1990.

15. Tavares-Dias M, Grandini AA, Prevalência e aspectos epidemiológicos de enteroparasitoses na população de São José da Bela Vista, SP, Revista da Sociedade Brasileira de Medicina Tropical 32: 63-65, 1999. 